\title{
MedDRA Low Level Term Code
}

National Cancer Institute

\section{Source}

National Cancer Institute. MedDRA Low Level Term Code. NCI Thesaurus. Code

C117048.

A coded value specifying the low level term from the Medical Dictionary for Regulatory Activities (MedDRA). 